Pacific Journal of Mathematics

TORSIONAL RIGIDITIES IN THE ELASTIC-PLASTIC
TORSION OF SIMPLY CONNECTED CYLINDRICAL BARS 


\title{
TORSIONAL RIGIDITIES IN THE ELASTIC-PLASTIC TORSION OF SIMPLY CONNECTED CYLINDRICAL BARS
}

\author{
Tsuan Wu TING
}

\begin{abstract}
Under elastic-plastic torsion, the circular shaft possesses the maximum resisting torque among all solid bars with the same cross-sectional area and the same angle of twist per unit length.
\end{abstract}

1. Introduction. Consider a simply connected cylindrical bar twisted by terminal couples. If the angle of twist per unit length is sufficiently small, then the bar behaves linearly elastic $[18,23,26]$. Under this circumstance, St. Venant succeeded in formulating it as a Neumann problem by means of his semi-inverse method, [22]. It was his contention that among all solid bars with the same crosssectional area, the circular shaft gives the maximum torsional rigidity. This isoperimetric problem was first solved by Pòlya [15]. Later similar results have also been obtained for multiply connected crosssections [17]. The results of Pòlya and Szegö have had much influence and further explorations of their problems have been continued up to the present time $[4,5,13-17,29]$.

According to the theory of plasticity [26, 27], if the angle of twist per unit length reaches a certain critical value, then some portion near the boundary of the bar becomes plastic. Moreover, the plastic region grows as the load increases, [26]. Although the elasticplastic torsion problem has been stated quite precisely for a long time, [28], the answers to the basic existence and regularity problems are recent ones, [2, 9, 11, 12, 26]. However, before the elastic-plastic torsion problem was completely settled, Leavitt and Ungar already showed that the circular shaft is also the strongest one under completely plastic torsion, [10].

Since the elastic-plastic torsion problem can be so formulated that it includes both the purely elastic and the completely plastic torsions as special cases, [26], it is the objective of this note to present a proof for the statement in the Abstract. Needless to say that Pòlya's ideas in his first and third proofs of St. Venant's conjecture will play an essential role in this proof. On the other hand, the present theorem includes Pòlya's results as well as the one obtained in [10].

2. The elastic-plastic torsion problem. Denote by $G$ the simply connected cross-section of a solid bar. We shall restrict $G$ to have the following properties: (i) $\partial G$, boundary of $G$, possesses continuously varying curvature except at a finite number of corners, 
(ii) between any two adjacent corners, the curvature of $\partial G$ achieves only a finite number of maxima and minima, and (iii) $G$ satisfies the well known cone conditions.

As usual, we denote by $C_{0}^{\infty}(G)$ the class of infinitely differentiable functions with compact support in $G$ and by $H_{0}^{1}(G)$ the completion of $C_{0}^{\infty}(G)$ under the Dirichlet norm. Let $\Psi$ be the distance function,

$$
\Psi(q)=k \rho(q, \partial G), q \in \bar{G} \equiv G+\partial G,
$$

where $k>0$ is the yielding constant and $\rho(q, \partial G)$ stands for the distance from $q$ to $\partial G$. Let $F$ be the closed convex subset of $H_{0}^{1}(G)$ specified by the rule:

$$
F=\left\{\varphi \mid \varphi \in H_{0}^{1}(G), \varphi \leqq \Psi \text { a.e. in } \bar{G}\right\},
$$

where $\Psi$ is the majorant function difined in (2.1). The elastic-plastic torsion problem is to find a function $\&$ in $F$ that minimizes the functional

$$
J[\varphi] \equiv \iint_{G}\left[|\operatorname{grad} \varphi|^{2}-4 \mu_{\theta} \varphi\right] d x d y
$$

among all $\varphi$ in $F$, where $\mu$ and $\theta$ are positive constants standing for the shear modulus and the angle of twist per unit length respectively.

Let $C^{n+\alpha}(G), 0<\alpha<1$, be the class of functions which together with its derivatives of order $n$ are Hölder continuous in $G$ with exponent $\alpha$. Then we have the following known results,

Theorem 1. The elastic-plastic torsion problem (2.3) has a unique solution $\psi$ belonging to $C^{1+\alpha}(G)$ such that wherever $\psi<\Psi, \psi$ is twice differentiable and satisfies the Poisson equation, $\Delta \psi=-2 \mu \theta$.

The existence and uniqueness of the minimizing extremal $\psi$ can be proved in an elementary way for the present case. The profound regularity result, $\psi \in C^{1+\alpha}(G)$, is essentially given in [2] and [11] and it has been carried out for the present case in [26] by establishing the existence of an elastic core.

There is another variational formulation for the same problem. It is to replace the admissible family $F$ by

$$
F_{1}=\{\varphi|| \operatorname{grad} \varphi \mid \leqq k \text { a.e. in } G\} \text {. }
$$

From the results in [26], it is easily seen that the two variational formulations are actually equivalent. However, a direct and essentially self-contained proof is given in [1].

For the convenience of later discussion we introduce some notations. Relative to the minimizing extremal $\psi$ and the majorant 
function $\Psi, \bar{G}$ can be partitioned as follows:

$$
E=\{q \mid q \in G, \psi(q)<\Psi(q)\}, P=\{q \mid q \in \bar{G}, \psi(q)=\Psi(q)\} .
$$

It turns out that $E$ is the elastic region and $P$ is the plastic region of $G$ as defined in plasticity theory, [18]. Let $q \in P$ and $s \in \partial G$ be such that $\rho(q, s)=\rho(q, \partial G)$. It is easy to check by using Theorem 1 that the line segment $q s$ lies in $P$. Using this result and Theorem 1 , we can show that $E$ is simply connected, [26].

3. Formulation of the isoperimetric problem. Given a simply connected domain $G$ with the specified properties, there is a unique minimizing extremal $\psi$ for the elastic-plastic torsion problem (2.3). Of course, $\psi$ depends upon the parameters $\mu$ and $\theta$. It depends on the parameter $k$ through the majorant function $\Psi$. If we keep the values of these three parameters fixed, then as the solution of the elastic-plastic torsion problem (2.3), $\psi$ is uniquely determined by the geometry of the domain $G$. Furthermore, to look into the effect of $G$, which is purely geometric in nature, we shall keep the area of $G$ fixed in the following discussions.

Relative to a rectangular Cartesian coordinate system with the $z$-axis parallel to the generators of the cylinder, the components of the Cauchy stress, [27], are given by

$$
t_{x x}=t_{y y}=t_{z z}=t_{x y}=0, t_{z y}=-\psi_{x}, t_{z x}=\psi_{y} .
$$

Hence, the resisting torque $M$ about the $z$-axis is given by

$$
\begin{aligned}
M(G) & \equiv \iint_{G}\left(x t_{z y}-y t_{x x}\right) d x d y=-\iint_{G}\left(x \psi_{x}+y \psi_{y}\right) d x d y \\
& =2 \iint_{G} \psi d x d y .
\end{aligned}
$$

Since for fixed values of the parameters $k, \theta$ and $\mu$ and for fixed area of $G, \psi$ is uniquely determined by the geometry of the domain $G$, the formula (3.1) shows that $M(G)$ is a functional defined on all simply connected domains $G$ with the same area and with the specified properties (i)-(iii). We wish to show that

THeorem 2. If $G$ is a simply connected domain with the same area as that of a disk $D$ and if it satisfies the conditions (i)-(iii) in the elastic-plastic torsion problem, then the domain functional $M$ defined in (3.1) must satisfy the inequality,

$$
M(G) \leqq M(D),
$$

where the equality sign holds when and only when $G$ is also a disk. 
Physically, the theorem means that among all solid bar with the same cross-sectional area and the same angle of twist, the circular shaft possesses the maximum resisting torque. Moreover, this is so no matter how large the angle of twist $\theta$ per unit length may be.

In order to cover the cases not included in linear elasticity theory we shall assume in the proof of Theorem 2 that the area of the plastic region $P$ in the disk $D$ is positive. It turns out the proof still holds even if the plastic region $P$ is empty.

It is true that the yielding of a circular shaft does not necessarily imply that all other shafts with the same cross-sectional area will also yield when twisted by the same amount. A simple example is a shaft with elliptic cross-sections. However, for some solid shafts, this is so. For example, a circular shaft with a circular groove along its generator. In order to cover these two possibilities in the proof of Theorem 2, we first establish a lemma which is also interesting by itself. Also, the results listed in the next section for the level curves of the extremal are essential for the Schwarz symmetrization used in the proof of Theorem 2 .

4. An auxiliary lemma. Consider the level curves of the minimizing extremal $\psi$,

$$
\psi(x, y)=\operatorname{const} \beta, 0<\beta<\max _{G+\partial G} \psi .
$$

It is quite clear that $\psi$ is nonnegative in $\bar{G}$ and hence the inequalities for specifying $\beta$ are meaningful. Each of such a level curve encloses an open subset $H(\beta)$ of $G$,

$$
H(\beta)=\{q \mid \psi(q)>\beta, q \in \bar{G}\} .
$$

For a general domain $G$ with the properties as was specified before, it is not known whether $H(\beta)$ is simply connected or even connected. However, the following facts are known:

(a) If $\partial H(\beta) \cap P$ is nonempty, then it consists of Jordan arcs which are either parallel to $\partial G$ or are circular arcs.

(b) All the Jordan arcs in $\partial H(\beta) \cap P$ possess continuously varying curvature.

(c) None of the boundary points of $\partial H(\beta) \cap P$ can be branch point, because they are interior points of $G$ and $|\operatorname{grad} \psi|=k$ there.

(d) Since $\psi$ satisfies the Poisson equation, $\Delta \psi=-2 \mu \theta$, in the elastic region $E$, it is analytic there and hence

$$
\partial H(\beta) \cap E=\partial H(\beta)-\partial H(\beta) \cap P
$$

consists of analytic curves. Consequently, the unique continuation 
theorem ensures that there are at most a finite number of points on $\partial H(\beta) \cap E$ at which $|\operatorname{grad} \psi|=0$.

(e) At each branch point on $\partial H(\beta)$ where $|\operatorname{grad} \psi|=0$, the level curve $\psi(x, y)=\beta$ has only a finite number of branches, [6].

The above results, (a)-(e), assure us that the open set $H(\beta)$ consists of only a finite number of components. Each of these components of $H(\beta)$ is enclosed by a simple closed curve with continuously varying tangents. With these facts in mind, we know that the Dirichlet problem,

$$
\Delta u=-2 \mu \theta \text { in } H(\beta), u=\psi \text { on } \partial H(\beta)
$$

always has a unique strict solution for $0<\beta<\max _{G} \psi$.

LEMma. If $u(x, y)$ is the solution of problem (4.3), then

$$
u(x, y) \geqq \psi(x, y) \text { everywhere in } H(\beta)
$$

and

$$
\iint_{H(\beta)} u(x, y) d x d y>\iint_{H(\beta)} \psi(x, y) d x d y
$$

unless $H(\beta) \cap P$ is empty.

Proof. If $H(\beta) \cap P$ is empty, then $u$ is identically equal to $\psi$ in $H(\beta)$ and there is nothing to prove. Accordingly, we assume that $H(\beta) \cap P$ is nonempty. The intersection property of the plastic region $P$ ensures that $H(\beta) \cap P$ consists of line segments perpendicular to $\partial G$. Moreover, $\partial P$ does not contain any segment perpendicular to $\partial G$. This fact can be proved by assuming the contrary and then by considering the Cauchy probem for $\psi_{x x}+\psi_{y y}=-2 \mu \theta$ with Cauchy date $\psi, \psi_{x}$, prescribed along a segment on the $y$-axis. The uniqueness theorem for the Cauchy problem will lead to a contradiction to the fact that $|\operatorname{grad} \psi|<k$ in $E$. These two properties of $P$ assure that if $H(\beta) \cap P$ is nonempty then it has positive area.

We proceed to show that if $u$ is the solution to (4.3) then

$$
u>\psi \text { a.e. in } H(\beta) \cap P \text {. }
$$

If this inequality has been established, it follows from the continuity of $u-\psi$ in $H(\beta) \cap P$ that $u \geqq \psi$ on $\partial(H(\beta) \cap P)$. Hence, an application of the maximum principle for elliptic inequalities [19] leads to that $u \geqq \psi$ on $H(\beta) \cap E$. This inequality together with the inequality (4.6) proves the lemma.

To establish (4.6) we first appeal to computation to check that 


$$
\Delta \psi=\Delta \psi=\frac{-k k(\mathrm{~s})}{[1-k(s) \tau]} \text { in } P-\partial P,
$$

where $s$ stands for the arc length of $\partial G, k$ the curvature of $\partial G$ and $\tau$ the distance measured from $\partial G$ along the inward normal. The expression on the right-hand side clearly shows that the set of points in $P$ on which $\Delta \psi=-2 \mu \theta$ always has two dimensional measure zero. Hence $u$ can not identically equal to $\psi$ in $H(\beta) \cap P$.

Suppose that $u \leqq \psi$ in $H(\beta) \cap P$. Then $u<\psi$ over some subset of positive area in $H(\beta) \cap P$. Moreover, it implies that $u \leqq \psi$ on $\partial(H(\beta) \cap E)$ and by maximum principle, $u \leqq \psi$ in $H(\beta) \cap E$. Consequently, $u \leqq \psi$ in $H(\beta)$ and

$$
\iint_{H(\beta)} u d x d y<\iint_{H(\beta)} \psi d x d y \text {. }
$$

That is, $u$ and $\psi$ can not be identical on $H(\beta)$. Now the Dirichlet principle for the solution $u$ to (4.3) implies the strict inequality,

$$
\begin{aligned}
& \iint_{H(\beta)}\left[|\operatorname{grad} u|^{2}-4 \mu \theta u\right] d x d y \\
& <\iint_{H(\beta)}\left[|\operatorname{grad} \psi|^{2}-4 \mu \theta \psi\right] d x d y .
\end{aligned}
$$

On the other hand, $u \leqq \psi$ on $H(\beta)$ implies that the function,

$$
\varphi \equiv \begin{cases}u & \text { in } H(\beta), \\ \psi & \text { in } \bar{G}-H(\beta),\end{cases}
$$

belongs to the admissible family $F$ for the elastic-plastic torsion problem. Hence, the minimizing property of $\psi$ implies that

$$
\begin{aligned}
& \iint_{H(\beta)}\left[|\operatorname{grad} \psi|^{2}-4 \mu \theta \psi\right] d x d y \\
& <\iint_{H(\beta)}\left[|\operatorname{grad} u|^{2}-4 \mu \theta u\right] d x d y .
\end{aligned}
$$

The contradiction caused by (4.7) and (4.8) proves that $u>\psi$ somewhere in $H(\beta) \cap P$ and that this set has positive area.

Let $H(\beta) \cap P_{1}$ be the maximum open subset of $H(\beta)$ over which $u>\psi$. If the set $H(\beta) \cap\left(P-P_{1}\right)$ has positive area, then we can apply the same reasoning for the set $H(\beta) \cap P$ to the set $H(\beta) \cap\left(P-P_{1}\right)$ and conclude that $u>\psi$ somewhere in $H(\beta) \cap\left(P-P_{1}\right)$. This contradicts the maximality of the set $H(\beta) \cap P_{1}$. Hence the assertion in (4.6) is established and the lemma is now proved.

In the proof of Theorem 2, we shall apply the lemma with $\beta>0$. Of course the lemma is true for the case $\beta=0$. In this case, $G(0)=G$ and the proof can be simplified. Hence we have the 
Corollary. Among two geometrically and elastically identical cylindrical bars, the one without yielding behaviors offers larger resisting torque under the same angle of twist. Accordingly, for safety in design the elastic-plastic theory is preferred.

5. Proof of Theorem 2. We shall compare the values of $M(G)$ with $M(D)$ by using the Schwarz symmetrization process [16, 21] to change the functions defined on $G+\partial G$ into functions defined on $D+\partial D$. To this end, let $B$ be the solid bounded by the domain $G$ and by the surface $z=\psi(x, y)$ for $(x, y)$ in $G+\partial G$, where $\psi$ is the miminizing extremal of the elastic-plastic torsion problem over $G$. Let $\Psi$ be the majorant function defined in (2.1). The Schwarz symmetrization is to change each section of the solid $B$ parallel to its base into a parallel disk with the same area. In this way, the solid $B$ is transformed into a solid $B^{*}$ of revolution with its base being the disk $D$ such that it is bounded from above by the surface of revolution $z=\psi^{*}(x, y)$ for $(x, y)$ in $D+\partial D$. The Schwarz symmetrization has the basic properties that it preserves volume and decreases surface area. More precisely, we have the Pòlya-Schwarz theorem,

$$
\begin{aligned}
I[\psi, G] & \equiv \iint_{G} \psi d x d y=\iint_{D} \psi^{*} d x d y \equiv I\left[\psi^{*}, D\right] \\
D[\psi, G] & \equiv \iint_{G}|\operatorname{grad} \psi|^{2} d x d y \geqq \iint_{G}\left|\operatorname{grad} \psi^{*}\right|^{2} d x d y \equiv D\left[\psi^{*}, D\right] .
\end{aligned}
$$

It should be mentioned that the regularity results for the solution to the elastic-plastic torsion problem assures that Schwarz's proof given in [21] can be applied here.

The above Schwarz symmetrization process can be applied to change the surface $z=\Psi(x, y),(x, y)$ in $G+\partial G$, into a surface $z=$ $\Psi^{*}(x, y),(x, y)$ in $D+\partial D$. Let $\Phi$ be the distance function, $\Phi(q)=$ $k \rho(q, \partial D)$ for all $q$ in $D$. We assert that

$$
\Phi(q) \geqq \Psi^{*}(q) \text { for all points } q \text { in } D,
$$

where the strict inequality holds everywhere in $D$ unless $G$ is also a disk. To show this we denote by $G(\rho)$ the set of all point in $G$ at distance $>\rho$ from $\partial G$. Let $S(\rho)$ be the total are length of $\partial G(\rho)$ and $A(\rho)$ the area of $G(\rho)$. Then we have the well-known isoperimetric inequality, $S(\rho) \geqq 2(\pi A(\rho))^{1 / 2}$. Consequently,

$$
-d A(\rho) \equiv S(\rho) d \rho \geqq 2(\pi A(\rho))^{1 / 2} d \rho,
$$

where the negative sign follows from the fact that $A(\rho)$ is a strictly decreasing function of $\rho$. Upon integrating the inequality from 0 to $\rho$, it yields 


$$
\rho \leqq \pi^{-1 / 2}\left[(A(0))^{1 / 2}-(A(\rho))^{1 / 2}\right] \equiv \rho^{*},
$$

where the equality sign holds only when $G$ is also a disk. Now for each $\rho \geqq 0, \Psi=k \rho$ on $\partial G(\rho)$. Moreover, under the Schwarz symmetrization $\Psi$ goes over to $\Psi^{*}$ and the region $G(\rho)$ enclosed by the level curve $\Psi \equiv k \rho$ changes into a disk $D\left(\rho^{*}\right)$ which has the same area as that of $G(\rho)$ and is bounded by the level curve $\Psi^{*}=k \rho$. Thus, $\Psi^{*}=k \rho$ on $\partial D\left(\rho^{*}\right)$. Hence for all $\rho^{*} \geqq 0$, we have from (5.3) that

$$
\Phi-\Psi^{*}=k\left(\rho^{*}-\rho\right) \geqq 0 \text { on } \partial D\left(\rho^{*}\right) .
$$

In fact, the equality sign in (5.4) holds only when either $\rho^{*}=0$ or $G$ is also a disk. This proves the assertion (5.2).

Let $\phi$ be the unique solution of the elastic-plastic torsion problem over the disk $D$. Then there is a unique constant $r_{0}$ such that

$$
\varphi=\left\{\begin{array}{l}
\mu \theta\left(r_{0}^{2}-r^{2}\right)+k\left(R-r_{0}\right), 0 \leqq r \leqq r_{0}, \\
k(R-r), r_{0} \leqq r \leqq R,
\end{array}\right.
$$

where $R$ is the radius of the disk $D$. Let $\rho_{0}^{*}=R-r_{0} . D\left(\rho_{0}^{*}\right)$ is the set of points in $D$ with distance $>\rho_{0}^{*}$ from $\partial D$. From the estimate in (5.2) and (5.5) we see that

$$
\varphi=\Phi \geqq \Psi^{*} \geqq \psi^{*} \quad \text { in } \quad D-D\left(\rho_{0}^{*}\right),
$$

where the first strict inequality sign holds everywhere in $D-D\left(\rho_{0}^{*}\right)$ unless $G$ is also a disk. As was already mentioned before, we assumed that $D-D\left(\rho_{0}^{*}\right)$ has positive area.

Let $v$ be the solution of the problem:

$$
\Delta v=-2 \mu \theta \text { in } D\left(\rho_{0}^{*}\right), v=\psi^{*} \quad \text { on } \partial D\left(\rho_{0}^{*}\right) \text {. }
$$

It follows immediately from (5.6), (5.7) and the maximum principle that

$$
\varphi-v=k r_{0}-\psi^{*}\left(r_{0}\right)>0 \text { in } D\left(\rho_{0}^{*}\right),
$$

unless $G$ is also a disk. From the very definition of Schwarz symmetrization of the solid $B$, we see that the region $H\left(\beta_{0}\right)$ in $G$, which is enclosed by the level curve

$$
\psi(x, y)=\beta_{0}=\psi^{*}\left(r_{0}\right)>0,
$$

is carried over onto the disk $D\left(\rho_{0}^{*}\right)$ under the Schwarz symmetrization. As were listed in $\S 4, \partial H\left(\beta_{0}\right)$ has all the nice properties, so we may consider the solution $u$ of the Dirichlet problem:

$$
\Delta u=-2 \mu \theta \text { in } H\left(\beta_{0}\right), u=\psi \text { on } \partial H\left(\beta_{0}\right) .
$$

According to the auxiliary lemma, 
$u \geqq \psi \quad$ everywhere in $H\left(\beta_{0}\right)$.

Under the Schwarz symmetrization, the function $u$ defined in (5.9) goes over to a function $u^{*}$ defined on $D\left(\rho_{0}^{*}\right)$ such that

$$
\begin{aligned}
I\left[u-\beta_{0}, H\left(\beta_{0}\right)\right] & =I\left[u^{*}-\beta_{0}, D\left(\rho_{0}^{*}\right)\right], \\
D\left[u-\beta_{0}, H\left(\beta_{0}\right)\right] & \geqq D\left[u^{*}-\beta_{0}, D\left(\rho_{0}^{*}\right)\right],
\end{aligned}
$$

where the notations for $I$ and $D$ are completely similar to that given in (5.1). Now, from (5.7) and from Pòlya's variational formulation for torsional rigidities [17], we see that

(5.12) $\quad I\left[v-\beta_{0}, D\left(\rho_{0}^{*}\right)\right]=\frac{I^{2}\left[v-\beta_{0}, D\left(\rho_{0}^{*}\right)\right]}{D\left[v-\beta_{0}, D\left(\rho_{0}^{*}\right)\right]} \geqq \frac{I^{2}\left[u^{*}-\beta_{0}, D\left(\rho_{0}^{*}\right)\right]}{D\left[u^{*}-\beta_{0}, D\left(\rho_{0}^{*}\right)\right]}$.

Also, it follows from (5.11) and (5.9) that

$$
\frac{I^{2}\left[u^{*}-\beta_{0}, D\left(\rho_{0}^{*}\right)\right]}{D\left[u^{*}-\beta_{0}, D\left(\rho_{0}^{*}\right)\right]} \geqq \frac{I^{2}\left[u-\beta_{0}, H\left(\beta_{0}\right)\right]}{D\left[u-\beta_{0}, H\left(\beta_{0}\right)\right]}=I\left[u-\beta_{0}, H\left(\beta_{0}\right)\right] .
$$

By combining (5.8), (5.12), (5.13), and (5.10), we find

$$
\begin{aligned}
I\left[\varphi-\beta_{0}, D\left(\rho_{0}^{*}\right)\right] & \geqq I\left[v-\beta_{0}, D\left(\rho_{0}^{*}\right)\right] \geqq I\left[u-\beta_{0}, H\left(\beta_{0}\right)\right] \\
& \geqq I\left[\psi-\beta_{0}, H\left(\beta_{0}\right)\right] .
\end{aligned}
$$

Since $D\left(\rho_{0}^{*}\right)$ and $H\left(\beta_{0}\right)$ have the same area, it follows immediately that

$$
I\left[\rho, D\left(\rho_{0}^{*}\right)\right] \geqq I\left[\psi, H\left(\beta_{0}\right)\right] \text {. }
$$

It may be noted that the above equality sign can not hold unless $H\left(\beta_{0}\right)$ is also a disk. On the other hand, the volume preserving property of Schwarz symmetrization implies that

$$
\begin{aligned}
& I\left[\psi^{*}, D-D\left(\rho_{0}^{*}\right)\right]+\beta_{0} \text { area of } D\left(\rho_{0}^{*}\right) \\
= & I\left[\psi, G-H\left(\beta_{0}\right)\right]+\beta_{0} \text { area of } H\left(\beta_{0}\right),
\end{aligned}
$$

and hence we have from this and the estimate in (5.6) that

$$
I\left[\varphi, D-D\left(\rho_{0}^{*}\right)\right] \geqq I\left[\psi^{*}, D-D\left(\rho_{0}^{*}\right)\right] \geqq I\left[\psi, G-H\left(\beta_{0}\right)\right],
$$

where the strict inequality sign holds unless either $D-D\left(\rho_{0}^{*}\right)$ is empty or $G$ is also a disk. By adding the corresponding sides in (5.14) and (5.15) we find

$$
I[\varphi, D] \geqq I[\psi, G]
$$

where the equality sign holds when and only when $G$ is a disk. Theorem 2 is now established. 


\section{REFERENCES}

1. H. R. Brezis and M. Sibony, Eqivalence de deux inequations variationnelles et applications, Arch. Rat. Mech. Anal. 41 (1971), 254-265.

2. H. R. Brezis and G. Stampacchia, Sur la régularité de la solution d'inequations elliptiques, Bull. Soc. Math. France, 96 (1968), 153-180.

3. H. Gajewski, Ein konstruktiver Existenz-und Einzigkeits-nachweis der Lösung des elastisch-plastischen Torsionproblems für prismatische Stäbe, Math. Nachs. 35 (1967), 153-169.

4. P. R. Garabedian, Proof of Uniqueness by Symmetrization, Studies in Analysis and Related Topics, Stanford University Press, Stanford, California, (1962), 126-127.

5. J. Hersch, Une interpretation du principe de Thomson et son analogue pour la frequence fondamentale d'une membrane, C. R. 248 (1959), 2062-62.

6. O. D. Kellogg, Foundation of Potential Theory, Frederick Unger, New York.

7. D. S. Kinderlehrer, The coincidence set of solutions of certain variational inequalities, Arch. Rat. Mech. Anal., 40 (1971), 231-250.

8. Helene Lanchon, Sur la solution du problème de torsion èlasto plastique d'une barre cylindrique de section multiconnexe, C. R. 271 serie A (1970), 1137-1140.

9. H. Lanchon and G. Duvant, Sur la solution du problème de la torsion èlasto plastique d'une barre cylindrique de section quelconque, C. R. Ser. A 264 (1967), 520-523.

10. Jay Leavitt and P. Ungar, Circle supports the largest sandpile, Comm. Pure Appl. Math., 15 (1962), 35-37.

11. H. Lewy and G. Stampacchia, On the regularity of the solution of a variational inequality, Comm. Pure Appl. Math., 22 (1969), 153-188.

12. - On existence and smoothness of solutions of some non-coercive variational inequalities, Arch. Rat. Mech. Anal., 41 (1971), 241-253.

13. E. Makai, On the principal frequency of a convex membrane and related problems, Czech. Math. J., 9 (1959), 66-70; Bounds for the principal frequency of a membrane and the torsional rigidity of a beam. Acta. Szeged, 20 (1959), 33-35.

14. L. E. Payne, Some Isoperimetric Inequalities in the Torsion Problem for Multiply Connected Regions, Studies in Analysis and Related Topics, Stanford Univ. Press, Stanford, California, (1962), 270-280.

15. G. Pòlya, Torsional rigidity, principal frequency, electro static capacity and symmetrization, Q. Appl. Math., 6 (1948), 267-277.

16. G. Pòlya and G. Szegö, Isoperimetric Inequalities in Mathematical Physics, Annals of Math. Studies, no. 27, Princeton Univ. Press, Princeton, N. J., 1951.

17. G. Pòlya and A. Weinstein, On the torsional rigidity of multiply connected crosssections, Annals Math., 52 (1950), 154-163.

18. W. Prager and P. G. Hodge, Jr., Theory of Perfectly Plastic Solids, John Wiley and Sons, New York, 1951.

19. M. H. Protter and H. F. Weinberger, Maximum Principle in Differential Equations, Prentice Hall, Englewood Cliffs, N. J., 1967.

20. - On the capacity of composite conductors, Math. Physics, 44 (1965), 375383.

21. H. A. Schwarz, Beweis des Sates, dass die Kugel k̇leinere Oberflache besizt, als jeder andere Körper gleichen Volumens, Gesammelte Mathematische Abhandlunger, Vol. 2, Verlag von Julius Springer, Berlin, (1890), 327-335.

22. B. De. St. Venant, Mémoire sur la torsion des prisms, Mem. div. sav. Acad. Sci., 14 (1856), 233-560.

23. T. Y. Thomas, Plastic Flow and Fracture in Solids, Academic Press, New York, 1961. 24. T. W. Ting, An isoperimetric inequality for moments of inertia of plane convex sets, Trans. Amer. Math. Soc., 107 (1963), 421-431.

25. - Upper and lower bounds of the radii of gyration of convex bodies. Trans. Amer. Math. Soc., 128 (1967), 336-357. 
26. T. W. Ting, Elastic-plastic torsion of simply connected cylindrical bars, I. U. Math. J., 20 (1971), 1047-1076.

27. C. Truesdell and W. Noll, The non-linear field theories of mechanics, Encyclopedia of Physics, III/3, Springer, New York, 1965.

28. R. Von Mises, Three remarks on the theory of the ideal plastic body, Reissner Ann. Volume, Edwards, Ann Arbor, Mich., (1949), 415-419.

29. H. F. Weinberger, Upper and lower bounds for torsional rigidities, J. Math. Phys., 32 (1953), 54-62.

Received February 4, 1972. Research partially supported by U. I. Research Council, and NSF Grant GP-33174X.

UNIVERSity of IllinoIs at URBana-Champaign, IllinoIS 



\section{PACIFIC JOURNAL OF MATHEMATICS}

\section{EDITORS}

D. Gilbarg and J. Milgram Stanford University

Stanford, California 94305

R. A. Beaumont

University of Washington

Seattle, Washington 98105
J. DugundJI

Department of Mathematics

University of Southern California

Los Angeles, California 90007

RICHARD ARENS

University of California

Los Angeles, California 90024

ASSOCIATE EDITORS
E. F. BECKENBACH
B. H. NeumanN
F. WOLF
K. YoSHIDA

\section{SUPPORTING INSTITUTIONS}

UNIVERSITY OF BRITISH COLUMBIA

CALIFORNIA INSTITUTE OF TECHNOLOGY

UNIVERSITY OF CALIFORNIA

MONTANA STATE UNIVERSITY

UNIVERSITY OF NEVADA

NEW MEXICO STATE UNIVERSITY

OREGON STATE UNIVERSITY

UNIVERSITY OF OREGON

OSAKA UNIVERSITY
UNIVERSITY OF SOUTHERN CALIFORNIA

STANFORD UNIVERSITY

UNIVERSITY OF TOKYO

UNIVERSITY OF UTAH

WASHINGTON STATE UNIVERSITY

UNIVERSITY OF WASHINGTON

$\stackrel{*}{*} \stackrel{*}{*} \stackrel{*}{*}$ AMERICAN MATHEMATICAL SOCIETY

NAVAL WEAPONS CENTER 


\section{Pacific Journal of Mathematics}

\section{Vol. 46, No. $1 \quad$ November, 1973}

Allan Francis Abrahamse, Uniform integrability of derivatives on $\sigma$-lattices .......................................... 1

Ronald Alter and K. K. Kubota, The diophantine equation $x^{2}+D=p^{n} \ldots \ldots \quad 11$

Grahame Bennett, Some inclusion theorems for sequence spaces .......... 17

William Cutler, On extending isotopies ........................ 31

Robert Jay Daverman, Factored codimension one cells in Euclidean

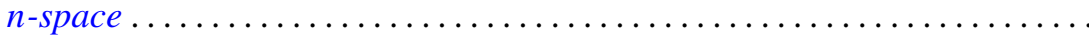

Patrick Barry Eberlein and Barrett O’Neill, Visibility manifolds ............ 45

M. Edelstein, Concerning dentability .......................... 111

Edward Graham Evans, Jr., Krull-Schmidt and cancellation over local

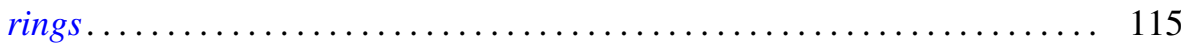

C. D. Feustel, A generalization of Kneser's conjecture ................ 123

Avner Friedman, Uniqueness for the Cauchy problem for degenerate parabolic equations .......................................... 131

David Golber, The cohomological description of a torus action ............ 149

Alain Goullet de Rugy, Un théorème du genre "Andô-Edwards" pour les

Fréchet ordonnés normaux..............................

Louise Hay, The class of recursively enumerable subsets of a recursively enumerable set ........................................

John Paul Helm, Albert Ronald da Silva Meyer and Paul Ruel Young, On orders of translations and enumerations...

Julien O. Hennefeld, A decomposition for $B(X)^{*}$ and unique Hahn-Banach

extensions

Gordon G. Johnson, Moment sequences in Hilbert space .

Thomas Rollin Kramer, A note on countably subparacompact spaces ...

Yves A. Lequain, Differential simplicity and extensions of a derivation ....

Peter Lorimer, A property of the groups Aut $\mathrm{PU}\left(3, q^{2}\right) \ldots$

225

Yasou Matsugu, The Levi problem for a product manifold.

231

John M.F. O'Connell, Real parts of uniform algebras ......

William Lindall Paschke, A factorable Banach algebra without bounded

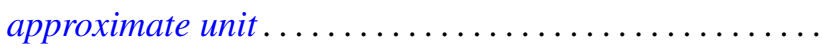

Ronald Joel Rudman, On the fundamental unit of a purely cubic field ....

Tsuan Wu Ting, Torsional rigidities in the elastic-plastic torsion of simply connected cylindrical bars .........................

Philip C. Tonne, Matrix representations for linear transformations on analytic sequences...................................

Jung-Hsien Tsai, On E-compact spaces and generalizations of perfect

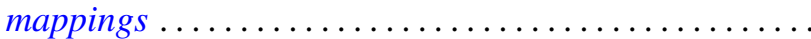

Alfons Van Daele, The upper envelope of invariant functionals majorized by an invariant weight. .. 